\title{
Quantum Tunneling in Mesoscopic Electromechanical Transducers
}

\author{
Nikita Pristupchik $^{1}$, Boris Konoplev ${ }^{1}$, Irina Kulikova ${ }^{1}$, Yulia Klunnikova ${ }^{1} \&$ Igor Lysenko $^{1}$ \\ ${ }^{1}$ Institute of Nanotechnology, Electronics and Electronic Equipment Engineering, The Department of Electronic \\ Apparatuses Design, Southern Federal University, Taganrog, Russia \\ Correspondence: Nikita Pristupchik, Institute of Nanotechnology, Electronics and Electronic Equipment \\ Engineering, The Department of Electronic Apparatuses Design, Southern Federal University, Taganrog, \\ Nekrasovsky st., 44, GSP-17A, Russia. Tel: 7-863-437-1603. E-mail: nkpristupchik@sfedu.ru
}

Received: September 17, 2014

doi:10.5539/mas.v9n2p20
Accepted: October 17, $2014 \quad$ Online Published: December 2, 2014

URL: http://dx.doi.org/10.5539/mas.v9n2p20

\begin{abstract}
The paper presents the simulation approach to some quantum transport effects, which arise in mesoscopic electromechanical transducers such as two-electrode tunneling transducer. The tunneling transducers are advanced nanoelectromechanical systems, which can be used for motion detection at nanoscale. It is very promising to adopt tunneling transducers as sensitive elements of the inertial microelectromechanical (or nanoelectromechanical) systems. In theory, their extremely high sensitivity is limited only by fundamental quantum relations. We propose theoretical model of the transducer based on the transfer-matrix formalism in this work. We provide the detailed derivation of transfer-matrix. Scattering potential of the transducer is a stepped representation of the electron potential energy. In addition, by using well-known formula proposed by L. Esaki and R. Tsu for finite superlattices we apply the numerical procedure for current density evaluation. The proposed model of the two-electrode mesoscopic tunneling transducer could be a useful tool for quantum transport simulation in high-performance nanoelectromechanical systems and advanced nanomaterials such as metal-polymer composites.
\end{abstract}

Keywords: micromachined inertial sensors, electromechanical displacement transducers, quantum tunneling, numerical simulation

\section{Introduction}

Electromechanical transducer is an essential part of any inertial microelectromechanical system (MEMS). Transducer converts proof mass displacements to the electrical signals. There are many types of the transducers based on different physical effects (Bocko \& Stephenson, 1991). Capacitive transducers are widely used in MEMS due to their flexibility and convenient microfabrication technology (Lysenko, 2013). However, these transducers have a lack of scalability. The displacements of the functional elements are negligible at nanoscale. Thus, corresponding capacity changing in transducer is very small and hard to detect. In addition, we should adopt comparatively complex service electronics to extract measurable signals out of capacitive transducers (Lysenko, Ryndin \& Dudin, 2012). On the other hand, tunneling transducers require low-noise interface circuits (Liu, Wen, Chen, Wen \& He, 2012).

One assumes that quantum-tunneling transducers could replace modern capacitive transducers in future because of their extremely high sensitivity at nanoscale (Bocko \& Stephenson, 1991; Gabrielson, 1993). Several designs for micromachined sensors augmented with quantum tunneling transducers have been reported (Patra \& Bhattacharyya, 2009; Shashkin et al., 2004; Vopilkin et al., 2012, 2014). It is notable, that resonant tunneling diodes may also be treated as quantum tunneling transducers (Xiong, Mao, Zhang, Wang, 2009; Zhang, Guo, Tang, Liu \& Wang, 2012).

Physical modeling of the MEMS devices augmented with quantum tunneling transducers is a challenging problem to solve because of its interdisciplinary nature (Bhattacharyya, Ghosh \& Paul, 2008; Konoplev, Pristupchik \& Ryndin, 2012; Pristupchik, Zaikin, Zavyalov \& Kosheleva, 2012). The main goal of our work is to provide a theoretical model of the mesoscopic two-electrode quantum-tunneling transducer based on transfer-matrix approach and tunneling current governing equation proposed by L. Esaki and R. Tsu (1973). This could be useful tool for quantum transport simulation in high-performance nanoelectromechanical systems. 
In addition, we discuss application of the model to the conductive polymer nanocomposites filled with metal particles such as quantum tunneling composite (QTC). The QTC is an elastic metal-polymer material with unusual properties (Bloor, Donnely, Hands, Laughlin \& Lussey, 2005; Bloor, Graham, Williams, Laughlin \& Lussey, 2006; Hands, Laughlin \& Bloor, 2012; Graham, Laughlin \& Bloor, 2013). QTC is able to change its resistance dramatically when a mechanical stress is applied. Thus, it is possible to produce high-efficiency electromechanical transducers made of QTC. One of the most interesting features of QTC is a non-linear current-voltage characteristic with hysteresis.

One states that unusual properties of this material can be explained involving electronic quantum transport through the tunneling connected metal particles chains. When particle spacing increases, the tunneling current is going down exponentially and vice-versa (Bloor et al., 2005, 2006; Hands et al., 2012; Graham et al., 2013). Thus, it is possible to state that conductive polymer is some kind of multi-electrode electromechanical transducer. The detailed theory has not been reported. We suppose that QTC can be simulated using model of the two-electrode mesoscopic transducer.

\section{Mesoscopic Electromechanical Transducers}

The schematic draft of a micromachined mesoscopic transducer is shown in Figure 1. The system consists of two identical electrodes, which can be moved relatively to each other, changing spatial gap between the electrodes $d_{L R}$. Bias voltage applied between right-hand side and left-hand side electrodes depicted as R and L respectively. A metal conductance of the electrodes is assumed. The isolating surface prevents leakage current other than direct quantum tunneling from emitter $(\mathrm{L})$ to collector $\mathbb{C}$.

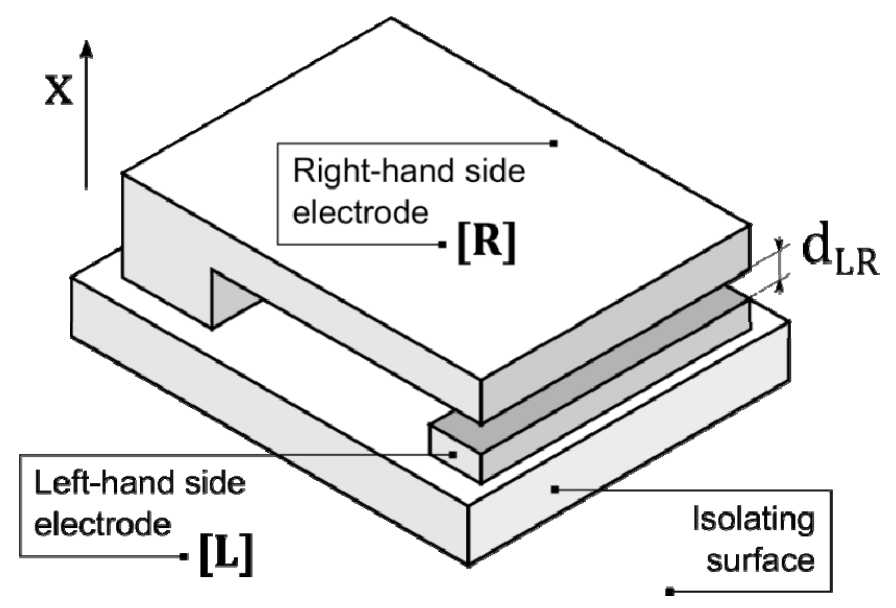

Figure 1. Two-electrode micromachined tunneling transducer

The external force applied to the right-hand side electrode along the $x$ direction will produce an elastic deformation of micromachined console and cause space gap $d_{L R}$ between the electrodes to change. It will change probability of quantum tunneling from $\mathrm{L}$ to $\mathrm{R}$ and corresponding current density. When external force is applied in the $x$ direction, current density will decrease and vice-versa.

Figure 2 presents multi-electrode mesoscopic transducer made of QTC. The system consists of several metal particles suspended in the polymer matrix. Particles can move relatively to each other, changing spatial gaps $d_{i j}$ between them. Bias voltage applied between right-hand side and left-hand side electrodes depicted as R and L respectively. We assume that direct quantum tunneling occurs between neighboring particles. Figure 2, a presents an initial state of the system. Figure 2, b illustrates how external force $F$ applied to the right-hand side electrode causes deformation of the elastic polymer matrix and reconfiguration of the particles. Left-hand side electrode is assumed to be fixed. Figure 2, $\mathrm{c}$ shows an equivalent circuit representation of the multi-electrode electromechanical transducer. 


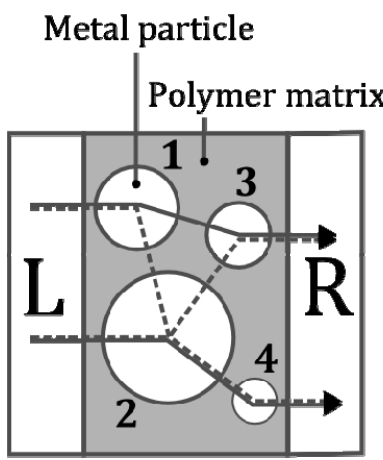

(a)

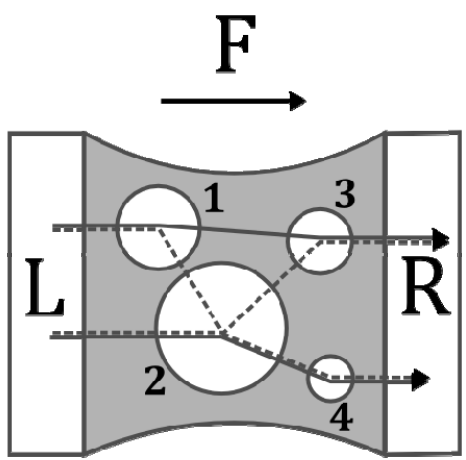

(b)

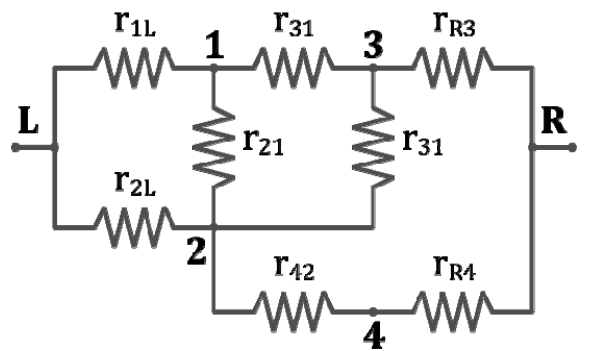

(c)

Figure 2. Multi-electrode QTC-based mesoscopic transducer. (a) Initial state of the multi-electrode transducer. (b) Stressed state of the transducer. (c) Equivalent electric scheme with lumped parameters

The proposed equivalent circuit is assumed to be quantitatively appropriate in extreme states of the QTC (insulating state and metal-conductivity state) when current-voltage characteristic of the material is linear. If we want to explain nonlinearity or hysteresis of the intermediate state, we should take to account additional physics associated with charging-discharging processes in dielectric media.

Applying the transfer-matrix method, we can calculate currents flowing in the circuit branches directly. Lumped parameters of the circuit should be recalculated every time when particle configuration is changed. It should be noted that topology of the circuit may also be changed due to a polymer matrix deformation. Thus, the equivalent circuit qualitatively illustrates how a theoretical model of the two-electrode mesoscopic transducer can be applied to the multi-electrode system.

\section{Quantum Transport Simulation}

To describe quantum transport in the two-electrode micromachined tunneling transducer one can use one-dimensional, steady-state Schrödinger equation (Konoplev et al., 2012; Pristupchik et al., 2012):

$$
-\frac{\hbar^{2}}{2 m_{0}} \frac{\partial^{2} \psi(x)}{\partial x^{2}}+U\left(x, \varphi_{w f}, d_{L R}, \Delta U\right) \psi(x)=\varepsilon \psi(x)
$$

where $\psi(x), m_{0}$ and $\varepsilon$ are the particle wavefunction, electron mass and energy, respectively, $\hbar$ is reduced Planck's constant and $U\left(x, \varphi_{w f}, d_{L R}, \Delta U\right)$ is the potential energy function shown in Figure 3 (solid line).

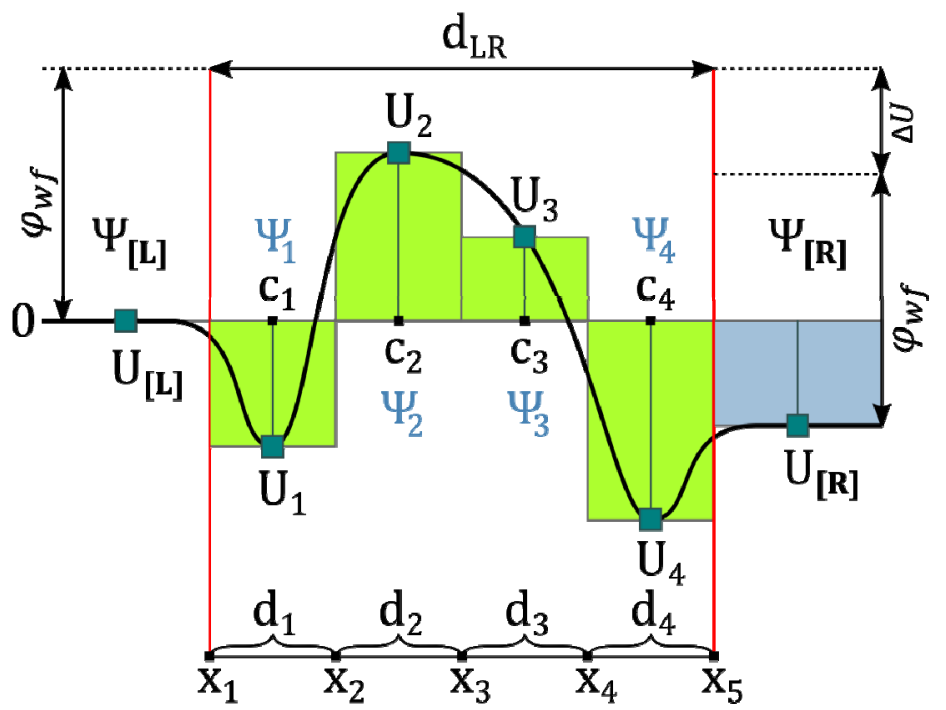

Figure 3. Layered representation of the generic scattering potential 
Spatial gap between the electrodes $d_{L R}$ is divided into layers $\left\{d_{j}\right\}$ in length, limited by grid points $\left\{x_{i}\right\}$. Conjugated grid $\left\{c_{j}\right\}$ is used to calculate stepped scattering potential $\left\{U_{j}\right\}$. Grid points $x_{1}$ and $x_{5}$ indicate emitter and collector surfaces respectively. Electrochemical potentials of the electrodes are $U_{L}$ and $U_{R}$ for emitter and collector respectively.

Discretization of the scattering potential allows writing down general solution of the equation (1) in form:

$$
\psi(x)=\left\{\begin{array}{c}
\psi_{L}(x)=A_{L}^{+} \exp \left(i \kappa_{L} x\right)+A_{L}^{-} \exp \left(-i \kappa_{L} x\right), \quad x<x_{1} \\
\psi_{j}(x)=A_{j}^{+} \exp \left(i \kappa_{j} x\right)+A_{j}^{-} \exp \left(-i \kappa_{j} x\right), \quad x \in\left[c_{i}-\frac{d_{i}}{2}, c_{i}+\frac{d_{i}}{2}\right] \\
\psi_{R}(x)=A_{R}^{+} \exp \left(i \kappa_{R} x\right)+A_{R}^{-} \exp \left(-i \kappa_{R} x\right), \quad x>x_{5}
\end{array}\right.
$$

where $\psi_{L}(x),\left\{\psi_{j}(x)\right\}$ and $\psi_{R}(x)$ are wavefunctions related to the corresponding layers shown in Figure 3; $A^{+}$and $A^{-}$are the amplitudes of the incident and reflected plane waves, respectively; $\kappa_{j}=\frac{1}{\hbar} \sqrt{2 m_{0}\left(\varepsilon-U_{j}\right)}$ is a local momentum; $\kappa_{L}=\frac{1}{\hbar} \sqrt{2 m_{0} \varepsilon}$ is a momentum corresponding to the left-hand side electrode; $\kappa_{R}=$ $\frac{1}{\hbar} \sqrt{2 m_{0}(\varepsilon+\Delta U)}$ is a momentum corresponding to the right-hand side electrode.

Applying appropriate boundary conditions at the layer interfaces, we can define how amplitudes transmit from the left side of the interface to the right side (Monsoriu, Villatoro, Marin, Urchueguia \& Cordoba, 2005):

$$
\begin{aligned}
& A_{L}^{+} \mathrm{e}^{i \kappa_{L} x_{1}}+A_{L}^{-} \mathrm{e}^{-i \kappa_{L} x_{1}}=A_{1}^{+} \mathrm{e}^{i \kappa_{1} x_{1}}+A_{1}^{-} \mathrm{e}^{-i \kappa_{1} x_{1}}, \\
& \kappa_{L} A_{L}^{+} \mathrm{e}^{i \kappa_{L} x_{1}}-\kappa_{L} A_{L}^{-} \mathrm{e}^{-i \kappa_{L} x_{1}}=\kappa_{1} A_{1}^{+} \mathrm{e}^{i \kappa_{1} x_{1}}-\kappa_{1} A_{1}^{-} \mathrm{e}^{-i \kappa_{1} x_{1}}, \\
& A_{j-1}^{+} \mathrm{e}^{i \kappa_{j-1} x_{j}}+A_{j-1}^{-} \mathrm{e}^{-i \kappa_{j-1} x_{j}}=A_{j}^{+} \mathrm{e}^{i \kappa_{j} x_{j}}+A_{j}^{-} \mathrm{e}^{-i \kappa_{j} x_{j}}, \\
& \kappa_{j-1} A_{4}^{+} \mathrm{e}^{i \kappa_{j-1} x_{j}}-\kappa_{4} A_{4}^{-} \mathrm{e}^{-i \kappa_{j-1} x_{j}}=\kappa_{j} A_{j}^{+} \mathrm{e}^{i \kappa_{j} x_{j}}-\kappa_{j} A_{j}^{-} \mathrm{e}^{-i \kappa_{j} x_{j}}, \\
& A_{4}^{+} \mathrm{e}^{i \kappa_{4} x_{5}}+A_{L}^{-} \mathrm{e}^{-i \kappa_{4} x_{5}}=A_{R}^{+} \mathrm{e}^{i \kappa_{R} x_{5}}+A_{R}^{-} \mathrm{e}^{-i \kappa_{R} x_{5}}, \\
& \kappa_{4} A_{4}^{+} \mathrm{e}^{i \kappa_{4} x_{5}}-\kappa_{4} A_{4}^{-} \mathrm{e}^{-i \kappa_{4} x_{5}}=\kappa_{R} A_{R}^{+} \mathrm{e}^{i \kappa_{R} x_{5}}-\kappa_{R} A_{R}^{-} \mathrm{e}^{-i \kappa_{R} x_{5}} .
\end{aligned}
$$

We can write down these equations in matrix notation as:

$$
\begin{gathered}
\left(\begin{array}{cc}
1 & 1 \\
\kappa_{L} & -\kappa_{L}
\end{array}\right) \times\left(\begin{array}{cc}
\mathrm{e}^{i \kappa_{L} x_{1}} & 0 \\
0 & \mathrm{e}^{-i \kappa_{L} x_{1}}
\end{array}\right) \times\left(\begin{array}{c}
A_{L}^{+} \\
A_{L}^{-}
\end{array}\right)=\left(\begin{array}{cc}
1 & 1 \\
\kappa_{1} & -\kappa_{1}
\end{array}\right) \times\left(\begin{array}{cc}
\mathrm{e}^{i \kappa_{1} x_{1}} & 0 \\
0 & \mathrm{e}^{-i \kappa_{1} x_{1}}
\end{array}\right) \times\left(\begin{array}{l}
A_{1}^{+} \\
A_{1}^{-}
\end{array}\right) \\
\left(\begin{array}{cc}
1 & 1 \\
\kappa_{j-1} & -\kappa_{j-1}
\end{array}\right) \times\left(\begin{array}{cc}
\mathrm{e}^{i \kappa_{j-1} x_{j}} & 0 \\
0 & \mathrm{e}^{-i \kappa_{j-1} x_{j}}
\end{array}\right) \times\left(\begin{array}{c}
A_{j-1}^{+} \\
A_{j-1}^{-}
\end{array}\right)=\left(\begin{array}{cc}
1 & 1 \\
\kappa_{j} & -\kappa_{j}
\end{array}\right) \times\left(\begin{array}{cc}
\mathrm{e}^{i \kappa_{j} x_{j}} & 0 \\
0 & \mathrm{e}^{-i \kappa_{j} x_{j}}
\end{array}\right) \times\left(\begin{array}{c}
A_{j}^{+} \\
A_{j}^{-}
\end{array}\right) \\
\left.\vdots \begin{array}{cc}
1 & 1 \\
\kappa_{4} & -\kappa_{4}
\end{array}\right) \times\left(\begin{array}{cc}
\mathrm{e}^{i \kappa_{4} x_{5}} & 0 \\
0 & \mathrm{e}^{-i \kappa_{4} x_{5}}
\end{array}\right) \times\left(\begin{array}{c}
A_{4}^{+} \\
A_{4}^{-}
\end{array}\right)=\left(\begin{array}{cc}
1 & 1 \\
\kappa_{R} & -\kappa_{R}
\end{array}\right) \times\left(\begin{array}{cc}
\mathrm{e}^{i \kappa_{R} x_{5}} & 0 \\
0 & \mathrm{e}^{-i \kappa_{R} x_{5}}
\end{array}\right) \times\left(\begin{array}{c}
A_{1}^{+} \\
A_{1}^{-}
\end{array}\right)
\end{gathered}
$$

For convenience, we can introduce special notation for used matrices and rewrite equation set (4) as:

$$
\begin{gathered}
\widehat{D}_{L} \widehat{K}_{x_{1}}^{l}\left|\chi_{L}\right\rangle=\widehat{D}_{1} \widehat{K}_{x_{1}}^{r}\left|\chi_{1}\right\rangle, \\
\vdots \\
\widehat{D}_{j-1} \widehat{K}_{x_{j}}^{l}\left|\chi_{j-1}\right\rangle=\widehat{D}_{j} \widehat{K}_{x_{j}}^{r}\left|\chi_{j}\right\rangle, \\
\vdots \\
\widehat{D}_{4} \widehat{K}_{x_{5}}^{l}\left|\chi_{4}\right\rangle=\widehat{D}_{R} \widehat{K}_{x_{5}}^{r}\left|\chi_{R}\right\rangle,
\end{gathered}
$$

where $\widehat{D}_{j-1}=\left(\begin{array}{cc}1 & 1 \\ \kappa_{j-1} & -\kappa_{j-1}\end{array}\right)$ is a discontinuous matrix, $\widehat{K}_{x_{j}}^{l}=\left(\begin{array}{cc}\mathrm{e}^{i \kappa_{j-1} x_{j}} & 0 \\ 0 & \mathrm{e}^{-i \kappa_{j-1} x_{j}}\end{array}\right)$ is a left-hand side phase matrix, $\widehat{K}_{x_{j}}^{r}=\left(\begin{array}{cc}\mathrm{e}^{i \kappa_{j} x_{j}} & 0 \\ 0 & \mathrm{e}^{-i \kappa_{j} x_{j}}\end{array}\right)$ is a right-hand side phase matrix, $\left|\chi_{j-1}\right\rangle=\left(\begin{array}{c}A_{j-1}^{+} \\ A_{j-1}^{-}\end{array}\right)$is a left-hand side state vector, $\left|\chi_{j}\right\rangle=\left(\begin{array}{c}A_{j}^{+} \\ A_{j}^{-}\end{array}\right)$is a right-hand side state vector.

One can resolve expressions of the equation set (5) relative to left-hand side state vectors, then these vectors can be recursively substituted to the corresponding equations as right-hand side state vectors, it gives: 


$$
\widehat{K}_{x_{1}}^{l}\left|\chi_{L}\right\rangle=\widehat{D}_{L}^{-1}\left[\prod_{j} \widehat{D}_{j} \hat{P}_{j} \widehat{D}_{j}^{-1}\right] \widehat{D}_{R} \widehat{K}_{x_{5}}^{r}\left|\chi_{R}\right\rangle,
$$

where $\hat{P}_{j}$ is a wave propagation matrix defined as:

$$
\hat{P}_{j}=\widehat{K}_{x_{i}}^{r}\left[\widehat{K}_{x_{i+1}}^{l}\right]^{-1}=\left(\begin{array}{cc}
\mathrm{e}^{-i \kappa_{j}\left(x_{i+1}-x_{i}\right)} & 0 \\
0 & \mathrm{e}^{i \kappa_{j}\left(x_{i+1}-x_{i}\right)}
\end{array}\right)=\left(\begin{array}{cc}
\mathrm{e}^{-i \kappa_{j} d_{j}} & 0 \\
0 & \mathrm{e}^{i \kappa_{j} d_{j}}
\end{array}\right),
$$

where $d_{j}=x_{i+1}-x_{i}$.

We can rewrite matrix equation (6) as:

$$
\left|\psi_{L}\right\rangle=\widehat{M}\left|\psi_{R}\right\rangle
$$

where $\left|\psi_{L}\right\rangle=\widehat{K}_{\chi_{1}}^{l}\left|\chi_{L}\right\rangle$ and $\left|\psi_{R}\right\rangle=\widehat{K}_{x_{5}}^{r}\left|\chi_{R}\right\rangle$ are redefined state vectors corresponding to wavefunctions of the emitter and collector, respectively; $\widehat{M}=\widehat{D}_{L}^{-1}\left[\prod_{j} \widehat{D}_{j} \widehat{P}_{j} \widehat{D}_{j}^{-1}\right] \widehat{D}_{R}$ is a transfer-matrix.

The initial state vector $\left|\psi_{L}\right\rangle$ corresponds to the particle incoming from the emitter. The final state vector $\left|\psi_{R}\right\rangle$ corresponds to the particle falling into the collector. If we take incident wavefunction amplitude as a measure for all the reflection and transmission amplitudes, and assume that there are no any scattering centers in the right-hand side electrode domain, we can write down:

$$
\left(\begin{array}{l}
1 \\
R
\end{array}\right)=\left(\begin{array}{ll}
M_{11} & M_{12} \\
M_{21} & M_{22}
\end{array}\right) \times\left(\begin{array}{l}
T \\
0
\end{array}\right)
$$

where $R$ is reflection amplitude (left-hand side electrode) and $T$ is transmission amplitude (right-hand side electrode). Tunneling particle can be in the reflected state or in the transmitted state, there are no other options, so we can write down (Feynman, Leighton \& Sands, 2013):

$$
R^{*} R+T^{*} T=|\boldsymbol{R}|^{2}+|T|^{2}=1 .
$$

If transfer-matrix elements are known, we can easily find out reflection amplitude from (9) and evaluate probability of transmission, which is commonly referred as transmission coefficient $T^{*} T$ :

$$
R=\frac{M_{21}}{M_{11}}, \quad T^{*} T=1-\frac{\left|M_{21}\right|^{2}}{\left|M_{11}\right|^{2}}
$$

Tunneling current density can be calculated by the formula (Tsu \& Esaki, 1973):

$$
J=\frac{|q| m_{0} k_{0} T_{0}}{2 \pi^{2} \hbar^{3}} \int_{0}^{\infty} T^{*} T \ln \left(\frac{1+\exp \left(-|q| \varepsilon / k_{0} T_{0}\right)}{1+\exp \left(-|q|(\varepsilon+\Delta U) / k_{0} T_{0}\right)}\right) d \varepsilon,
$$

where $k_{0}$ is Boltzmann constant, $T_{0}$ is absolute temperature, $\varepsilon$ is energy measured from the Fermi level of the emitter.

It is possible to integrate equation (12) numerically if transmission function values are tabulated. Performing the integration for different bias voltages and distances, one can get a family of current-voltage characteristics. Numerical integration procedure involves several steps.

First, we need to construct a continuous scattering potential parameterized with bias voltage $\Delta U$, work function of the electrodes $\varphi_{w f}$ and distance between the electrodes $d$. The detailed description of scattering potential is presented in Appendix A. When imaging force is neglected, the potential is:

$$
U(x)=\left\{\begin{array}{cl}
0, & x \leq 0 \\
\varphi_{w f}-\frac{\Delta U}{d} x, & 0<x \leq d . \\
-\Delta U, & x>d
\end{array}\right.
$$

Second step is generation of the basic coordinate grid $X_{b}=\left\{x_{i} \mid i=\overline{1, n}, x_{1}=0, x_{n}=d\right\}$. Grid steps in most general case can be expressed as $\Delta x_{i}=x_{i+1}-x_{i}$. Thus, spatial gap between the electrodes is sliced by layers with lengths

$\Delta_{j}=\Delta x_{i}$. We use $j$ for layer indexation. Also we need to generate a conjugated coordinate grid $X_{c}=$ $\left\{\xi_{j} \mid j=\overline{1, n-1}\right\}$, where $\xi_{1}=\frac{1}{2} \Delta x_{1}$, and $\xi_{n-1}=d-\frac{1}{2} \Delta x_{n-1}$. Conjugated coordinate grid step is $\Delta \xi_{j}=\frac{1}{2}\left(\Delta_{j}+\Delta_{j-1}\right)$. 
Next, we have to construct stepped scattering potential using conjugated coordinate grid $X_{c}$. In most general form, stepped scattering potential could be written down as:

$$
\begin{gathered}
V(x)=\sum_{j} U\left(\xi_{j}\right) \cdot f_{j} ; \\
f_{j}(x)=\left\{\begin{array}{l}
0, x \notin\left(\xi_{j}-\frac{\Delta_{j}}{2}, \xi_{j}+\frac{\Delta_{j}}{2}\right) \\
1, x \in\left[\xi_{j}-\frac{\Delta_{j}}{2}, \xi_{j}+\frac{\Delta_{j}}{2}\right]
\end{array} .\right.
\end{gathered}
$$

Stepped scattering potential $V(x)$ is a continuous representation of the $\left\{U_{j}\right\}$ set, which is used to define local wavefunctions in (2).

The tunneling particle energy spectrum is continuous. Transfer matrix have to be constructed for each allowed energy value. Thus, direct solution of the described problem is impossible due to infinite number of allowed states and infinite number of corresponding transfer matrices.

We can discretize continuous energy spectrum setting limits and defining energy grid $E_{b}=\left\{\epsilon_{k} \mid k=\overline{1, m}\right\}$, where limits are $\epsilon_{1}=0$, and numerical calculations show that $\epsilon_{n}=2 \varphi_{w f}$ is a suitable estimation. The grid step is $\Delta \epsilon_{k}=\epsilon_{k+1}-\epsilon_{k}$. Thus, allowed energy range is divided by intervals $\delta_{l}=\Delta \epsilon_{k}=\vec{\delta}$. We use $l$ for interval indexation. Also we need to generate conjugated energy grid $E_{c}=\left\{\varepsilon_{l} \mid l=\overline{1, m-1}\right\}$, where $\varepsilon_{1}=\frac{1}{2} \Delta \epsilon_{1}$, and $\varepsilon_{m-1}=2 \varphi_{w f}-\frac{1}{2} \Delta \epsilon_{k-1}$. The conjugated energy grid points are used to characterize energy of tunneling electrons.

Next, we can construct transfer matrices as previously described and evaluate transmission coefficients for each energy state from $E_{c}$ set. We can also represent expression under integral sign in (12) as vector:

$$
\vec{F}=\left\{T_{l}^{*} T_{l} \cdot \ln \frac{1+\exp \left(-|q| \varepsilon_{l} / k_{0} T_{0}\right)}{1+\exp \left(-|q|\left(\varepsilon_{l}+\Delta U\right) / k_{0} T_{0}\right)} \mid l=\overline{1, m-1}\right\},
$$

where $T_{l}^{*} T_{l}$ is a transmission coefficient for energy $\varepsilon_{l}$. Taking that into account, we can replace formula (12) proposed by L. Esaki and R. Tsu (1973) with expression:

$$
J=\frac{|q| m_{0} k_{0} T_{0}}{2 \pi^{2} \hbar^{3}} \times(\vec{\delta} \cdot \vec{F}),
$$

where integral was replaced by inner production of vectors $\vec{F}$ and $\vec{\delta}$. Expression (16) allows computing tunneling current density numerically. Parameters of the discretization are basic coordinate grid points number $n$ and basic energy grid points number $m$. These parameters affects solution accuracy and time consumption. Thus, they should be properly chosen, and that is feasible concern of the future work.

\section{Results and Discussion}

Figure 4 shows that current-voltage characteristics obtained with transfer-matrix method (numerical solution) are in good agreement (for low-voltages) with characteristics obtained with generalized formula for tunneling current (analytical solution) proposed by Simmons (1963). Simmons has obtained his formula in case of quasi-classical approximation proposed by Wentzel-Kramers-Brillouin (WKB). It is necessary to emphasize that characteristics are equivalent up to the constant scaling factor. We assume that analytical solution should be scaled because of WKB limitations. It is well known that WKB-approximation in quantum transport case is only appropriate when a transmission coefficient is much smaller than 1 (Landau \& Liphshitz, 1973). In our case, there are no any reasons to suspect transmission coefficient to be small (Konoplev et al., 2012; Pristupchik et al., 2012). The disagreement between the analytical and numerical solutions increases with higher bias voltage values. Explanation of this phenomenon requires more studies and does not present in this paper.

The generalized formula for tunneling current has been recently used in papers (Vopilkin et al., 2012, 2014) as suitable approximation for quantum transport simulation in tunneling transducer of a MEMS tunneling sensor, because of good agreement with the experiment. The analytical expression is appropriate to describe current-voltage characteristics of the tunneling transducers up to the constant scaling factor only (Vopilkin et al., 2012). 


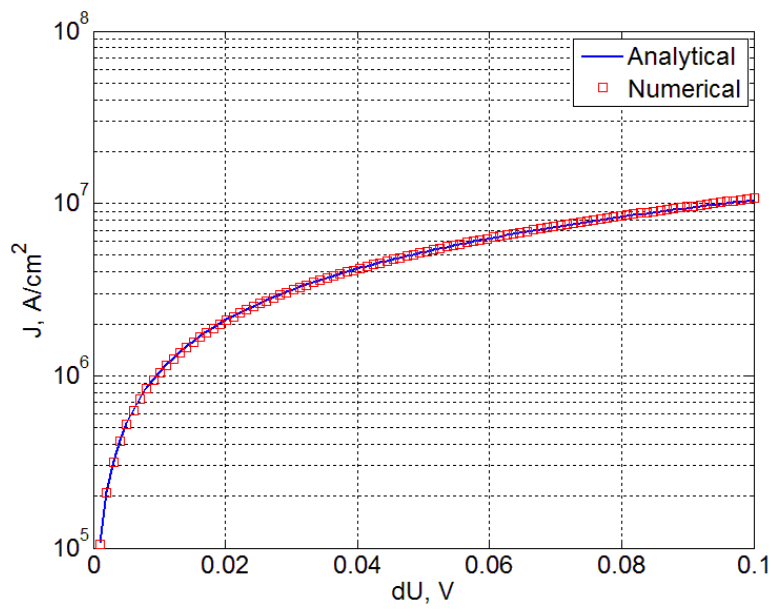

(a)

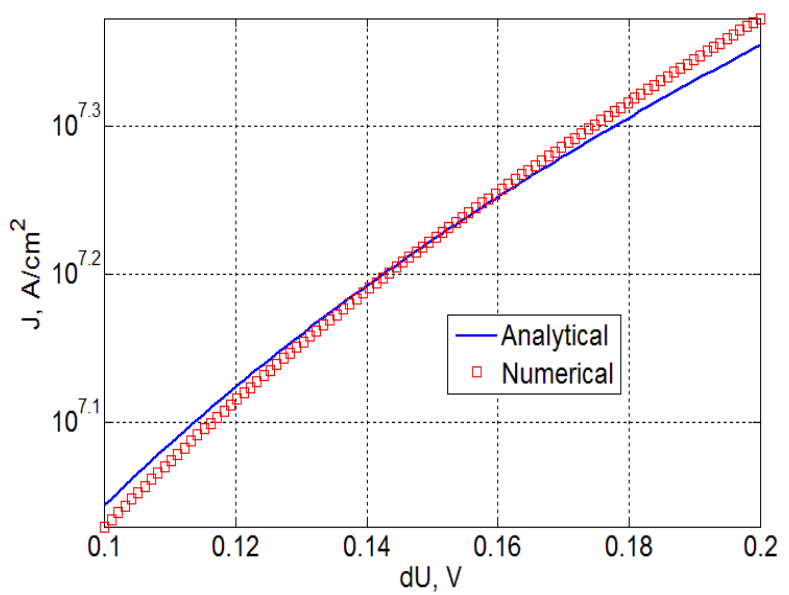

(b)

Figure 4. Analytical and numerical current-voltage characteristics comparison. (a) Bias voltage varies in range from $0 \mathrm{mkV}$ to $100 \mathrm{mkV}$. (b) Bias voltage varies in range from $100 \mathrm{mkV}$ to $200 \mathrm{mkV}$

The main advantage of transfer-matrix approach in case of quantum transport is flexibility. The scattering potential can vary in broad range without any restrictions. Thus, it is possible to state that presented method is good enough for variety of practical calculations. The proposed numerical procedure is simple enough to implement a computational algorithm using e.g. MATLAB-system embedded language.

The main limitation of the presented numerical method is computation expensiveness for realistic scattering potentials. The time consumption is comparatively high and it is a result of numerous matrix multiplications (one propagation matrix and two discontinuity matrices for each layer of scattering potential). Regardless of time expense, memory consumption is low due to small ranks of the matrices. Thus, comparatively simple reliefs can be studied on entry-level hardware.

\section{Conclusions and Further Work}

In this paper, we have studied the simulation approach to quantum transport in mesoscopic electromechanical transducers such as two-electrode tunneling transducer. We have presented quantum-level theoretical model of the transducer using transfer-matrix method. We have provide detailed derivation of the transfer-matrix. In addition, by using well-known formula proposed by L. Esaki and R. Tsu (1973) for finite superlattices we apply a numerical procedure for current density evaluation, which involves space and energy domain discretization.

The proposed model of the two-electrode mesoscopic tunneling transducer could be a useful tool for quantum transport simulation in high-performance nanoelectromechanical systems and advanced nanomaterials such as metal-polymer composites. Thus, one can generalize method described above to perform first-principle simulation of the metal-polymer composites similar to QTC.

\section{Acknowledgements}

This study was supported by the Ministry of education and science of Russia (project no. 213.01-11/2014-12, project no. 14.575.21.0045, RFMEFI57514X0045) and the Russian foundation for basic research (project no. 13-07-00274).

\section{References}

Bhattacharyya, T. K., Ghosh, A., \& Paul, D. (12-14 February 2008). Physical modelling of a MEMS based electron tunneling accelerometer. 2008 IEEE Sensors Applications Symposium, SAS-2008 - Proceedings, IEEE, 101-106.

Bloor, D., Donnely, K., Hands, P. J., Laughlin, P., \& Lussey, D. (2005). A metal-polymer composite with unusual properties. Journal of Physics D: Applied Physics, 38, 2851-2860, http://dx.doi.org/10.1088/0022-3727/38/16/018 
Bloor, D., Graham, A., Williams, E. J., Laughlin, P. J., \& Lussey, D. (2006). Metal-polymer composite with nanostructured filler particles and amplified physical properties. Applied Physical Letters, 88, http://dx.doi.org/10.1063/1.2183359

Bocko, M. F., \& Stephenson, K. A. (1991). Tunneling transducers: Quantum limited displacement monitors at the nanometer scale. Journal of Vacuum Science and Technology B: Microelectronics and Nanometer Structures, 9, 1363-1366, http://dx.doi.org/10.1116/1.585197

Feynman, R., Leighton, R., \& Sands, M. (2013). The Feynman lectures on physics. Retrieved from http://www.feynmanlectures.caltech.edu/III_13.html

Gabrielson, T. B. (1993). Mechanical-thermal noise in micromachined acoustic and vibration sensors. IEEE Transactions on Electron Devices, 40(5), 903-908, http://dx.doi.org/10.1109/16.210197

Graham, A., Laughlin, P. J., \& Bloor, D. (2013). Metal-polymer composite sensors for volatile organic compounds: Part 2. Stand alone chemi-resistors. Sensors and Actuators, B: Chemical, 177, 507-514. http://dx.doi.org/10.1016/j.snb.2012.11.046

Hands, P. J., Laughlin, P. J., \& Bloor, D. (2012). Metal-polymer composite sensors for volatile organic compounds: Part 1. Flow-through chemi-resistors. Sensors and Actuators B: Chemical, 162, 400-408, http://dx.doi.org/10.1016/j.snb.2011.12.016

Konoplev, B. G., Pristupchik, N. K., \& Ryndin, E. A. (2012). Displacement transducers based on tunneling effect simulation method. Vestnik SSC RAS (in Russian), 4, 20-26.

Landau, L. D., \& Lifshitz, E. M. (1977). Quantum mechanics: Non-relativistic theory (3d ed.). New York, NY: Pergamon.

Liu, H., Wen, Z., Chen, L., Wen, Z., \& He, X. (2012). A novel low noise interface ASIC of vacuum microelectronic accelerometer. Chinese Journal of Sensors and Actuators, 25, 911-916, http://dx.doi.org/10.3969/j.issn.1004-1699.2012.07.009

Lysenko, I. E. (2013). Functionally integrated micro- and nanomechanical angular rates and linear accelerations sensors. Taganrog: SFedU.

Lysenko, I. E. (2013). Modeling of the micromachined angular rate and linear acceleration sensor LL-type with redirect of drive and sense axes. World Applied Sciences Journal, 27, 759-762, http://dx.doi.org/10.5829/idosi.wasj.2013.27.06.13703

Lysenko, I. E., Ryndin, E. A., \& Dudin, N. K. (2012). Signal processing devices of micromechanical components capacitor converters.Journal of Nano and Microsystem Technique (in Russian), 7, 48-51.

Monsoriu, J. A., Villatoro, F. R., Marin, M. J., Urchueguia, J. F., \& Cordoba P. F. (2005). A transfer matrix method for the analysis of fractal quantum potentials. European Journal of Physics, 26, 603-610, http://dx.doi.org/10.1088/0143-0807/26/4/005

Patra, S., \& Bhattacharyyaa, T. K. (2009). High sensitive surface micromachined out of plane tunneling accelerometers with low-g resolution. IEEE/ASME International Conference on Advanced Intelligent Mechatronics, AIM, IEEE/ASME, 1577-1581, http://dx.doi.org/10.1109/AIM.2009.5229848

Pristupchik, N. K., Zaikin, A. N., Zavyalov, D. K., \& Kosheleva, N. N. (2012). Modeling method of tunneling displacement transducers of MEMS. Fundamental Research (in Russian), 11(3), 640-644.

Shashkin, V. I., Vostokov, N. V., Vopilkin, E. A., Klimov, A. Y., Volgunov, D. G., Rogov, V. V., \& Lazarev, S. G. (2004). High-sensitivity accelerometer based on cold emission principle. IEEE Sensors Journal, 4, 211-215. http://dx.doi.org/10.1109/JSEN.2004.823683

Simmons, J. G. (1963). Generalized formula for the electric tunnel effect between similar electrodes separated by a thin insulating film. Journal of Applied Physics, 34(6), 1793-1803. http://dx.doi.org/10.1063/1.1702682

Tsu, R., \& Esaki, L. (1973). Tunneling in a finite superlattice. Applied Physics Letters, 22, 562-564, http://dx.doi.org/10.1063/1.1654509

Vopilkin, E. A., Klimov, A. Yu., Rogov, V. V., Pryakhin, D. A., Gusev, S. A., Skorohodov, E. V., ... Shashkin, V. I. (2014). MEMS tunneling sensor without the feedback loop. IEEE Sensors Journal, 14(6), 1831-1835. http://dx.doi.org/10.1109/JSEN.2014.2305307 
Vopilkin, E. A., Klimov, A. Y., Rogov, V. V., Shuleshova, I. Y., Pryahin, D. A., Gusev, S. A., ... Shashkin, V. I. (2012). MEMS tunneling accelerometer sensor. Journal of Nano and Microsystem Technique (in Russian), $5,48-53$.

Xiong, J. J., Mao, H. Y., Zhang, W. D., \& Wang, K. Q. (2009). A novel micro-accelerometer with adjustable sensitivity based on resonant tunnelling diodes. Chinese Physics B, 18, 1242-1247, http://dx.doi.org/10.1088/1674-1056/18/3/067

Zhang, B., Guo, H., Tang, J., Liu, J., \& Wang, J. (2012). Temperature characteristic optimizations of the RTD sensitivity for the MEMS sensor applications. Applied Mechanics and Materials, ADME 2012, 1906-1910, http://dx.doi.org/10.4028/www.scientific.net/AMM.220-223.1906

\section{Appendix A}

\section{Scattering Potential with Image Force Included}

When electrochemical potentials of the electrodes without bias are taken to be equivalent (workfunctions are equal), potential energy of the two-electrode electromechanical transducer can be expressed in the form:

$$
U\left(x, \varphi_{w f}, d_{L R}, \Delta U\right)=U_{G}\left(x, \varphi_{w f}, d_{L R}\right)+U_{C}\left(x, \varphi_{w f}, d_{L R}\right)+U_{B}\left(x, d_{L R}, \Delta U\right) .
$$

The first term $U_{G}$ in (A1) is rectangular potential barrier. The spatial gap between $\mathrm{L}$ and $\mathrm{R}$ referred as $d_{L R}$. The second term $U_{C}$ is correlation energy associated with the image force applied to the tunneling electron. The third term is bias energy function $U_{B}$ caused by bias voltage $\Delta U$ applied to the electrodes. Cartesian coordinate is $x$. The work function of the electrodes is $\varphi_{w f}$. Thus, the potential energy $U$ is a function of the coordinate $x$ parameterized with $\Delta U, d_{L R}$ and $\varphi_{w f}$.

The $U_{G}$ term can be expressed as:

$$
\begin{gathered}
U_{G}\left(x, \varphi_{w f}, d_{L R}\right)=\varphi_{w f} \cdot W\left(x, d_{L R}\right) ; \\
W\left(x, d_{L R}\right)=\left\{\begin{array}{l}
0, x \notin\left(-\frac{d_{L R}}{2}, \frac{d_{L R}}{2}\right) \\
1, x \in\left[-\frac{d_{L R}}{2}, \frac{d_{L R}}{2}\right]
\end{array}\right.
\end{gathered}
$$

It is assumed that in a solid unoccupied electronic state corresponds to the presence of a positive charge. One can refine the scattering potential, incorporating the potential energy of interaction of the tunneling electron and the field of two point positive charges, one of which is on the surface of the emitter, and the other one is on the surface of the collector:

$$
U_{C}\left(x, \varphi_{w f}, d_{L R}\right)=U_{C L}\left(x, \varphi_{w f}, d_{L R}\right)+U_{C R}\left(x, \varphi_{w f}, d_{L R}\right),
$$

where $U_{C L}\left(x, \varphi_{w f}, d_{L R}\right)$ is correction function, which expresses the interaction of the electron with the surface of the emitter; $U_{C R}\left(x, \varphi_{w f}, d_{L R}\right)$ is correction function, which expresses the interaction of the electron with the surface of the collector.

Correction functions $U_{C L}\left(x, \varphi_{w f}, d_{L R}\right)$ and $U_{C R}\left(x, \varphi_{w f}, d_{L R}\right)$ can be expressed as:

$$
\begin{aligned}
& U_{C L}\left(x, \varphi_{w f}, d_{L R}\right)=\left\{\begin{array}{rl}
-\frac{|q|^{2}}{4 \pi \varepsilon_{0}\left(x+\frac{d_{L R}}{2}\right)+\frac{|q|^{2}}{\varphi_{w f}}}, & x \notin\left(-\frac{d_{L R}}{2},+\infty\right) \\
0, & x \in\left(-\infty,-\frac{d_{L R}}{2}\right]
\end{array},\right. \\
& U_{C R}\left(x, \varphi_{w f}, d_{L R}\right)=\left\{\begin{array}{r}
\frac{|q|^{2}}{4 \pi \varepsilon_{0}\left(x-\frac{d_{L R}}{2}\right)-\frac{|q|^{2}}{\varphi_{w f}}}, x \notin\left(-\infty,+\frac{d_{L R}}{2}\right) \\
0, x \in\left[\frac{d_{L R}}{2},+\infty\right)
\end{array},\right.
\end{aligned}
$$

where $q$ is electron charge; $\varepsilon_{0}$ is permittivity of free space. It is assumed that there are no charge carriers in the gap between the electrodes.

Expression (A5) is constructed so that the action of the induced positive charge on the surface of the emitter applied in the direction of the collector. Function (A6), in contrast, allows for the action of the positively charged vacancy on the surface of the collector.

Assuming the bias electric field is completely confined in the gap, bias energy function $U_{B}\left(x, d_{L R}, \Delta U\right)$ is: 


$$
U_{B}\left(x, d_{L R}, \Delta U\right)=\left\{\begin{array}{c}
0, x \leq 0 \\
\varphi_{w f}-\frac{\Delta U}{d} x, 0<x \leq d \\
-\Delta U, x>d
\end{array}\right.
$$

\section{Copyrights}

Copyright for this article is retained by the author(s), with first publication rights granted to the journal.

This is an open-access article distributed under the terms and conditions of the Creative Commons Attribution license (http://creativecommons.org/licenses/by/3.0/). 